\title{
Efeito da cobertura de dossel sobre a comunidade de Odonata (insecta) em igarapés na região de Santarém-Belterra (PA)
}

\begin{abstract}
Ações antrópicas, como a remoção da vegetação ripária, causam prejuízos ao homem e à natureza; essa, quando integrada, exerce grande influência positiva sobre o corpo d'água; quando retirada, pode causar alterações físicas e bióticas sobre os ecossistemas aquáticos e os indivíduos que nela habitam. Neste contexto, foi avaliado o efeito da cobertura de dossel sobre a comunidade de Odonata na região Santarém-Belterra, Pará, Brasil. Para o estudo, foram amostrados 48 igarapés, estes que, para suas classificações, quanto aos níveis de conservação, foi aplicado o Índice de Integridade do Habitat. Para a amostragem biológica, os cursos foram medidos por uma distância de $100 \mathrm{~m}$ ao longo de sua extensão, subdivididos em 20 segmentos de $5 \mathrm{~m}$ cada, os Odonatas adultos foram capturados por uma rede entomológica durante 3 minutos em cada seguimento. Através de IIH, classificamos 26 igarapés, com integridade alterada e 22 preservadas. Foram coletados 1819 insetos, sendo 365 da subordem Anisoptera e 1454 de Zygoptera. Os valores obtidos através regressão linear apontaram que, com o aumento de aproximadamente $0,2 \mathrm{de} \mathrm{IIH}$, ocorre um decréscimo de aproximadamente oito indivíduos e uma espécie de Anisoptera e aumento de aproximadamente sete indivíduos e uma espécie de Zygoptera. As maiores riquezas de espécies de Anisoptera ocorreram em igarapés classificados como alterados ( $\mathrm{t}=2,072 ; \mathrm{p}=0,043)$, para Zygoptera, este índice foi maior em igarapés classificados como preservados $(t=2,387 ; p=0,021)$. Com aumento de aproximadamente $10 \%$ na cobertura de dossel, há um decréscimo de aproximadamente três indivíduos e uma espécie de Anisoptera e um aumento de aproximadamente dois indivíduos e uma espécie de Zygoptera com o mesmo percentual. A diferença na distribuição dos indivíduos da ordem Odonata pode ser explicada por suas exigências ecofisiológicas inerentes a cada subordem. Onde a vegetação ripária exerce maior influência sobre a riqueza das espécies. Dada a especificidade de cada subordem, elas podem ser utilizadas como bioindicadores de qualidade do hábitat.
\end{abstract}

Palavras-chave: Anisoptera; Zygoptera; Abundância, Riqueza; Dossel.

\section{Effect of canopy cover on Odonata (insecta) community in streams in Santarém-Belterra (PA)}

\begin{abstract}
Anthropic actions, such as the removal of riparian vegetation, cause damage to man and nature; This, when integrated, has a great positive influence on the body of water; when withdrawn, it can cause physical and biotic changes to aquatic ecosystems and their inhabitants. In this context, the effect of canopy cover on the Odonata community in the Santarém-Belterra region, Pará, Brazil was evaluated. For the study, 48 streams were sampled, which, for their classification, regarding the conservation levels, was applied the Habitat Integrity Index. For biological sampling, the courses were measured at a distance of $100 \mathrm{~m}$ along their length, subdivided into 20 segments of $5 \mathrm{~m}$ each, the adult Odonatas were captured by an entomological net for 3 minutes in each follow-up. Through IIH, we classified 26 streams, with altered integrity and 22 preserved. 1819 insects were collected, 365 from the suborder Anisoptera and 1454 from Zygoptera. The values obtained through linear regression indicated that, with the increase of approximately $0.2 \mathrm{of} \mathrm{IIH}$, there is a decrease of approximately eight individuals and one species of Anisoptera and increase of approximately seven individuals and one species of Zygoptera. The highest richness of Anisoptera species occurred in streams classified as altered $(t=2.072 ; p=0.043)$, for Zygoptera, this index was higher in preserved streams $(t=2.387 ; p=0.021)$. With an approximately $10 \%$ increase in canopy coverage, there is a decrease of approximately three individuals and one species of Anisoptera and an increase of approximately two individuals and one species of Zygoptera with the same percentage. The difference in the distribution of individuals of the order Odonata can be explained by their inherent ecophysiological demands on each suborder. Where riparian vegetation has the greatest influence on species richness. Given the specificity of each suborder, they can be used as habitat quality bioindicators.
\end{abstract}

Keywords: Anisoptera; Zygoptera; Abundance, Wealth; Canopy.

Topic: Conservação da Biodiversidade

Reviewed anonymously in the process of blind peer.
Received: 09/08/2018

Approved: 17/08/2018

Daniel de Carvalho Silva

Universidade Federal do Oeste Pará, Brasil

http://lattes.cnpq.br/1960500948326519

daniel14dcs@gmail.com

José Max Barbosa Oliveira Junior (ib

Universidade Federal do Oeste do Pará, Brasil

http://lattes.cnpq.br/1353014365045558

http://orcid.org/0000-0002-0689-205X

maxbio@hotmail.com

Referencing this:

SILVA, D. C.; OLIVEIRA JUNIOR, J. M. B.. Efeito da cobertura de dossel sobre a comunidade de Odonata (insecta) em igarapés na região de Santarém-Belterra (PA). Revista Ibero-Americana de Ciências Ambientais, v.9, n.6, p.88-97, 2018. DOI:

http://doi.org/10.6008/CBPC2179-6858.2018.006.0011 


\section{INTRODUÇÃO}

Mata ciliar, também conhecida como mata ripária ou ripícola, é toda a vegetação presente na margem de corpos hídricos, sejam rios, riachos ou lagoas, e que apresentam em sua composição árvores ou arbustos (GALVÃO, 2000). Este tipo de ambiente possui grande valor por apresentar heterogeneidade, com grande variedade de espécies, o que oferece maior abundância com relação a outras constituições florestais; proporcionando a propagação de vegetação por facilitar a passagem para imigração de espécies (FIRMINO, 2003), serve como uma barreira para amparar o curso d'água, possibilitando servir como habitat para a fauna silvestre e para não elevar a temperatura da água (VALENTE et al., 2005).

Ações antrópicas como a remoção da vegetação ripária causa numerosos prejuízos ao homem e a natureza, pois a mesma, quando íntegra, exerce grande influência positiva sobre o corpo d'água (GALVÃO, 2000), quando retirada pode causar alterações físicas e bióticas sobre os ecossistemas aquáticos (ANGERMEIER et al., 1994), levando a problemas como o aumento crescente da sedimentação, alteração grave do ciclo hidrológico e pode ainda afetar os parâmetros físico-químicos da água disponível (CALLISTO et al., 2001; POND et al., 2008), que são nocivos a sua integridade; logo, alteram a biodiversidade local (ANGERMEIER et al., 1994).

Neste contexto, surge como forma de mesurar problemas que podem ocorrer por fatores naturais ou antropogênicos nos ambientes, o biomonitoramento, que se trata de uma atividade que visa o estudo das respostas de organismos vivos, chamados de bioindicadores, para avaliar as mudanças nesses ambientes (MATTHEWS et al., 1982). Os bioindicadores são organismos cujo suas funções vitais estão totalmente correlacionadas a fatores ambientais (CALLISTO et al., 2006), estes que, quando o meio em que habitam está sofrendo ações prejudiciais à sua integridade, respondem através de seu comportamento (MATSUURA, 2000).

As libélulas (Insecta: Odonata), são insetos que possuem um ciclo de vida longo, podendo alcançar até dois anos; predadores encontrados em corpos hídricos de água doce e no ecossistema aquático variam de local dependendo do seu estágio de vida (MARCO, 1998; FERREIRA-ROMERO, 1998). Gorham et al. (1992) aponta que, dependendo da espécie, pode haver uma variação quanto à tolerância a certos ambientes, ou seja, uma espécie pode ser mais tolerante e outra mais sensível às alterações a qual o local é submetido. Por estas características, os Odonata são fortemente cotados como bioindicadoras de alterações em ecossistemas aquáticos (MARCO, 1998; FERREIRA-ROMERO, 1998).

A ordem Odonata apresenta três subordens (FERREIRA-PERUQUETE, 2004; SOUZA et al., 2007): Anisoptera, cujo seus indivíduos possuem asas posteriores mais largas que as anteriores e os olhos unidos; Zygoptera, que possuem olhos separados, a base das asas semelhantes e pousam com as asas sobrepostas sobre o dorso; e Anizygoptera (não existente no Brasil) (FERREIRA-PERUQUETE, 2004). Em contexto nacional compõe 14 famílias, sendo 10 da ordem Zygoptera e 4 de Anisoptera (CORBET et al., 1995), que segundo Clausnitzer et al. (2009), uma a cada 10 espécies se encontram ameaçadas de extinção por conta das ações humanas. 
Apesar de demonstrarem extrema sensibilidade a alterações em seu habitat (SANTOS et al., 1998), ainda há carência de estudos do efeito das ações antrópicas nos ecossistemas aquáticos, sobre a fauna de Odonata (PERUQUETTI et al., 2002). Com isso, faz-se necessário o incentivo dos mesmos, pois por suas características fisiológicas e ecológicas (CORBET, 1983), este grupo apresenta excelência como bioindicador de qualidade de ambientes aquáticos por relacionar perturbações ambientais (SANTOS et al., 1998).

Portanto, este estudo possui grande relevância, tendo em vista que estes são necessários para que ações preventivas ou remediadoras sejam aplicadas de forma efetiva para a conservação e manutenção da estrutura física do ambiente e da biodiversidade local (VEECH et al., 2002). Diante do exposto, o trabalho objetivou testar o efeito da cobertura de dossel sobre a comunidade de Odonata (Insecta) em pequenos igarapés na região de Santarém/Belterra (PA).

\section{MATERIAIS E MÉTODOS}

\section{Área De Estudo}

O estudo foi realizado em 48 igarapés da região Santarém-Belterra, Oeste do estado do Pará, norte do Brasil, conforme proposto na figura 1. De acordo com a classificação de Köppen, o clima predominante na região é classificado como $A m w$, caracterizado como tropical chuvoso. A região possui temperatura média anual de $25,5^{\circ} \mathrm{C}$, precipitação acumulada anual de $1983 \mathrm{~mm} /$ ano, com o período de menor disponibilidade de água, que vai do mês de julho a dezembro, com o mês de setembro estimado como o mais seco, com precipitação de 35,02mm. A vegetação local é classificada como Floresta Tropical Densa, Floresta Tropical Aberta e Floresta Alterada (ESPÍRITO-SANTO, 2003).

\section{Amostragem Biológica}

Os 48 igarapés amostrados foram medidos por uma distância de $100 \mathrm{~m}$ ao longo de sua extensão, subdivididos em 20 segmentos de $5 \mathrm{~m}$ cada. As amostragens foram realizadas em sequência durante três minutos em cada seguimento, totalizando uma hora de amostragem em cada igarapé. Os Odonata adultos foram capturados com uso de uma rede entomológica com diâmetro de $40 \mathrm{~cm}$ e profundidade de $65 \mathrm{~cm}$, tendo como suporte um cabo de alumínio de 90cm (OLIVEIRA-JUNIOR et al., 2015).

Estudos em regiões neotropicais apontam que a presença de indivíduos de Odonata, sejam larvas ou adultos, são encontradas em maior densidade no período de estiagem (BAPTISTA et al., 2001; FULAN et al., 2007). Portanto, as coletas ocorreram em período de estiagem (entre os meses de julho e agosto de 2010). As amostras foram coletadas quando a radiação solar atingiu seu pico (das $10 \mathrm{~h}$ às $14 \mathrm{~h}$ ), para garantir que todos os grupos de Odonata (conformadores térmicos, heliotérmicos e endotérmicos) estivessem ativos no momento das coletas (MARCO et al., 2002). Este método tem sido utilizado com sucesso em outros estudos, pois a divisão em seguimento faz com que as áreas não fiquem saturadas com a retirada dos indivíduos (MARCO, 1998; OLIVEIRA-JUNIOR et al., 2015).

A identificação taxonômica foi realizada no Laboratório Multidisciplinar do curso de Gestão Ambiental da Universidade Federal do Oeste do Pará, Pará, Brasil, para tal foram utilizadas chaves 
especializadas (BORROR, 1945; BELLE, 1988, 1996; GARRISON, 1990; LENCIONI, 2005; GARRISON et al., 2006, 2010). Após a identificação, os espécimes de insetos foram depositados no laboratório supracitado.

\section{Análise da integridade ambiental dos igarapés}

Para avaliar a integridade dos igarapés, foi utilizado o Índice de Integridade de Habitat (IIH) proposto por Nessimian et al. (2008). A avaliação deste protocolo foi realizada por um único amostrador para reduzir variações. Este método empregou 12 parâmetros que avaliaram: O uso da terra próximo ao corpo d'água; Largura da mata ciliar e seu estado de conservação; Condição da mata ciliar por uma distância de 10m; Tamanho do substrato; Estrutura e erosão dos barrancos; vegetação aquática e detritos; e mesohabitat (corredeiras, piscinas profundas, meandros) presentes. Cada questão deste questionário continha de 4 a 6 alternativas, dispostas a representar as variações na qualidade do ambiente. $O$ índice final varia em uma escala de 0 a 1, e quanto mais próximo for de 1, mais integro é esse sistema (PEREIRA et al., 2012; MONTEIROJÚNIOR et al., 2013, 2014; GIEHL et al., 2014).

\section{Cobertura de dossel e Análise dos dados}

Medidas da cobertura do dossel no canal foram obtidas com uso de um densiômetro. Tal medida foi realizada seis vezes em cada segmento: centro direito, centro esquerda, centro montante, centro jusante, e próximo às margens direita e esquerda. A medida final de cada igarapé foi a média dessas medidas. Para avaliar o efeito da integridade dos igarapés sobre a abundância e riqueza de espécies, foram realizadas regressões lineares simples, pois através desta análise foi possível obter uma relação funcional entre as variáveis (ZAR, 1999).

Para testar a diferença na abundância e riqueza de espécies entre as categorias de conservação (alterado e preservado), foi realizado teste T de Student (ZAR, 2008). Para avaliar o efeito da cobertura de dossel sobre a abundância e riqueza de espécies de Anisoptera e Zygoptera também foram realizadas regressões lineares simples (ZAR, 1999). Todas as análises foram realizadas pelas rotinas do programa $R$ Development Core Team 2011, utilizando o pacote vegan.

\section{DISCUSSÃO TEÓRICA}

\section{Índice de Integridade do Habitat e Descrição da Comunidade de Odonata}

Os valores obtidos através da aplicação do Índice de Integridade do Habitat (IIH) determinou que dos 48 igarapés amostrados, 26 apresentavam integridade alterada (IIH variando de 0,08 a 0,69), enquanto, 22 foram classificados como preservados (IIH variando de 0,70 a 0,99). Para o presente estudo, foram coletados 1.819 exemplares de Odonata, distribuídos em 10 famílias, 33 gêneros e 67 espécies. A subordem Anisoptera apresentou 365 exemplares, identificados em duas famílias (Libellulidae e Gomphidae), 16 gêneros e 36 espécies, enquanto Zygoptera apresentou 1454 exemplares, oito famílias (Calopterygidae, Coenagrionidae, Dicteriadidae, Megapodagrionidae, Perilestidae, Polythoridae, Protoneuridae e Pseudostigmatidae) 17 gêneros e 32 espécies. 
Para Anisoptera, Libellulidae foi a família com maior abundância (358), e os gêneros Erythodiplax $(n=193)$, Orthemis $(n=35)$ e Oligoclada $(n=31)$ foram as mais abundantes dentro dessa família. Para Zygoptera, Calopterygidae constituiu a família mais abundante com 505 espécimes; Mnesarete $(n=372)$, Chacolpteryx $(n=213)$ e Argia $(n=208)$ foram os gêneros mais abundantes dessa família.

\section{Efeito da Integridade Ambiental Sobre a Abundância e Riqueza de Espécies}

O Índice de Integridade do Hábitat $(\mathrm{IIH})$ afeta tanto a abundância $\left(r^{2}=0,409 ; p<0,001\right)$, conforme se vê na figura 1; quanto à riqueza de espécies de Anisoptera $\left(r^{2}=0,346 ; p<0,001\right)$, como se pode notar na figura 1B. O aumento da integridade ambiental gera um decréscimo na abundância e riqueza de espécies de Anisoptera. Com o aumento de aproximadamente $0,2 \mathrm{de} \mathrm{IIH,} \mathrm{há} \mathrm{um} \mathrm{decréscimo} \mathrm{de} \mathrm{aproximadamente} \mathrm{oito}$ indivíduos e uma espécie de Anisoptera. Também foi observado efeito do IIH sobre abundância $\left(r^{2}=0,180\right.$; $p=0,003$ ) (figura 1C) e riqueza de espécies de Zygoptera $\left(r^{2}=0,268 ; p<0,001\right)$, proposto na figura 1D. 0 aumento da integridade ambiental gera um aumento na abundância e riqueza de espécies de Zygoptera. Com o aumento de aproximadamente 0,2 de IIH, há um aumento de aproximadamente sete indivíduos e uma espécie de Zygoptera.
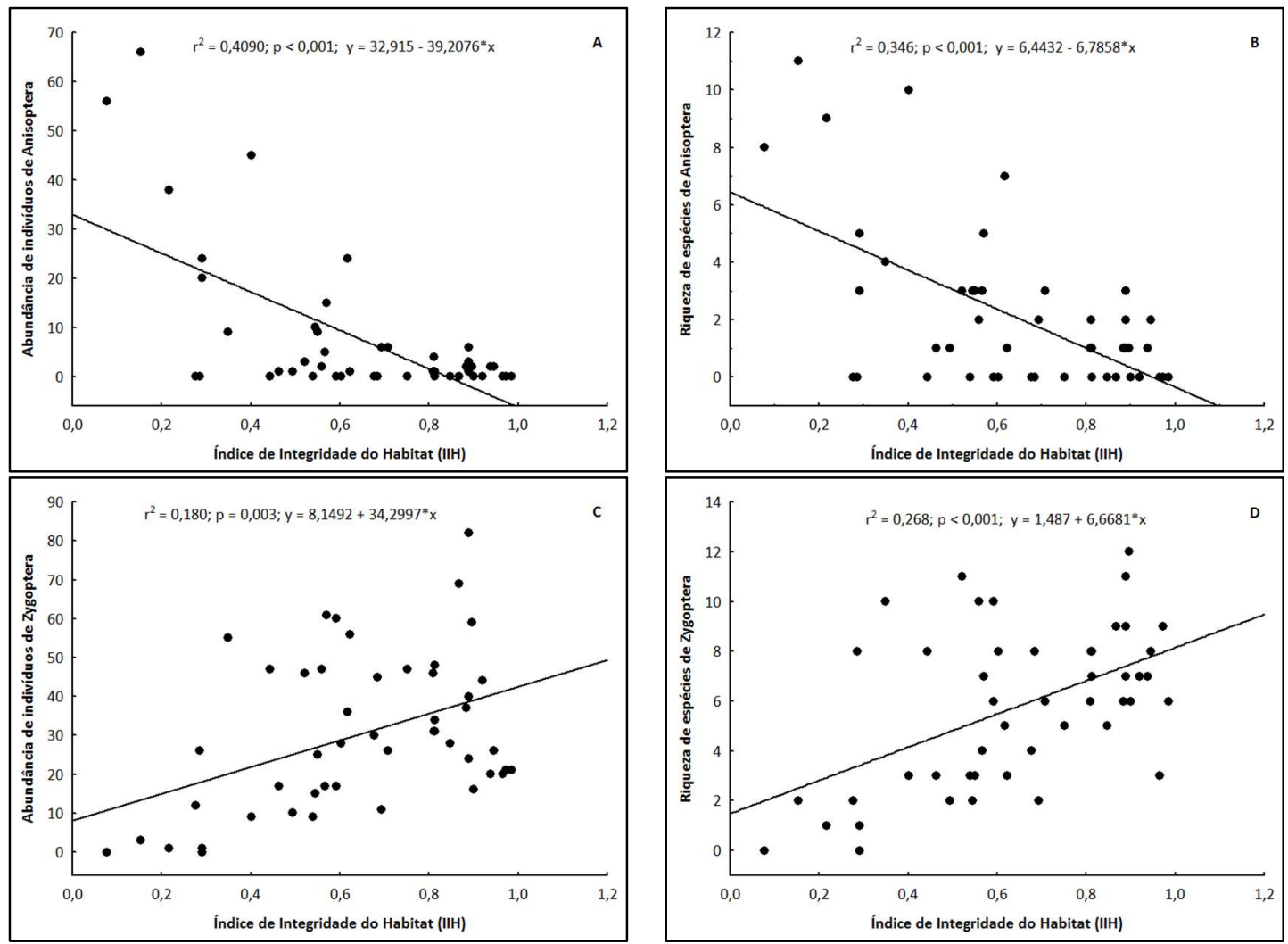

Figura 1: A) Relação entre abundância; B) riqueza de espécies de Anisoptera; C) abundância; D) riqueza de espécies de Zygoptera e a integridade ambiental (Índice de Integridade do Habitat) em igarapés amostrados na região SantarémBelterra (PA).

As maiores riquezas de espécies de Anisoptera ocorreram em igarapés classificados como alterados $(t=2,072 ; p=0,043)$. Esses ambientes apresentaram em média duas espécies a mais do que os ambientes preservados (figura 2A); para Zygoptera, este índice foi maior em igarapés classificados como preservados 
$(t=2,387 ; p=0,021)$, estes ambientes apresentam em média três espécies a mais do que os ambientes alterados, conforme se pode ver na figura $2 \mathrm{~B}$.
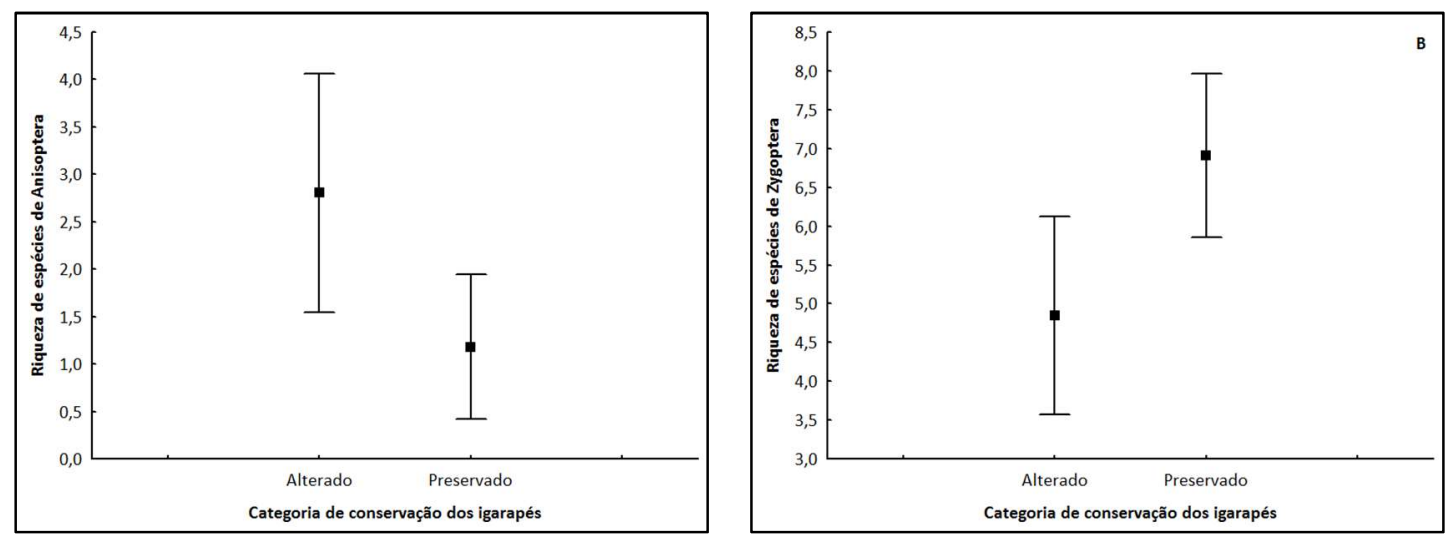

Figura 2: A) Riqueza de espécies de Anisoptera; e B) Zygoptera, amostrados em igarapés com diferentes níveis de conservação na região Santarém-Belterra (PA).

\section{Efeito da Cobertura de Dossel Sobre Abundância e Riqueza de Espécies}

A cobertura de dossel afeta tanto a abundância $\left(r^{2}=0.333, p<0,001\right)$ (figura $\left.3 A\right)$, quanto a riqueza de espécies de Anisoptera $\left(r^{2}=0.336, p<0,001\right)$, ilustrado na figura 3B. O aumento da cobertura de dossel gera um decréscimo na abundância e riqueza de espécies de Anisoptera. Com o aumento de aproximadamente 10\% na cobertura de dossel há um decréscimo de aproximadamente três indivíduos e uma espécie de Anisoptera.
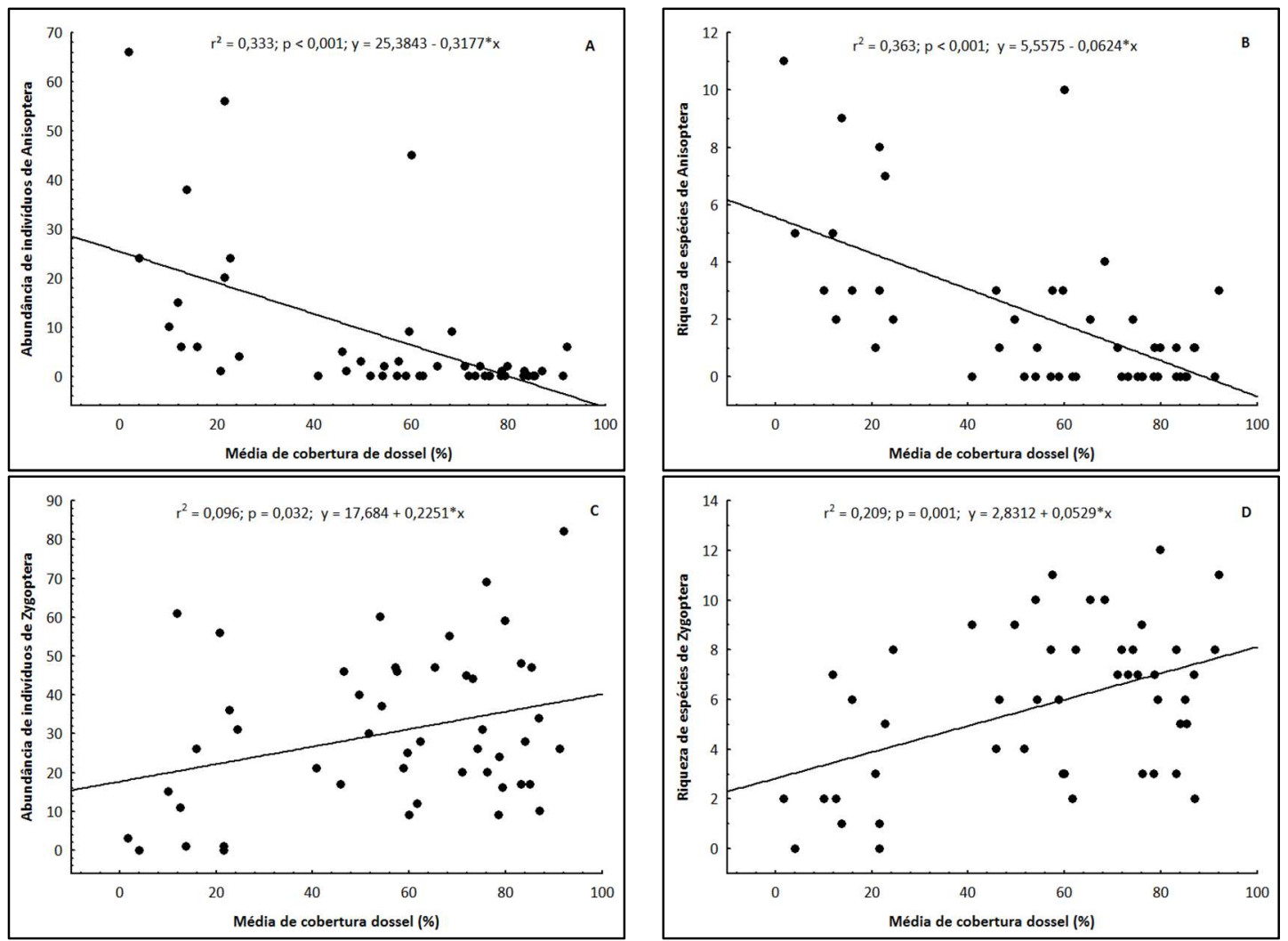

Figura 3: A) Relação entre média de cobertura de dossel (\%) e abundância; e B) riqueza de espécies de Anisoptera; C) abundância; e riqueza de espécies; (D) de Zygoptera em igarapés amostrados na região Santarém-Belterra (PA). 
Também foi observado efeito da cobertura de dossel sobre abundância $\left(r^{2}=0,096, p<0,032\right)$, proposto na figura $3 C$ e riqueza de espécies de Zygoptera $\left(r^{2}=0.209, p=0.001\right)$, conforme se pode notar na figura 3D. 0 aumento da cobertura de dossel gera um aumento na abundância e riqueza de espécies de Zygoptera. Com o aumento de aproximadamente $10 \%$ na cobertura de dossel há um aumento de aproximadamente dois indivíduos e uma espécie de Zygoptera. Dadas as exigências ecofisiológicas de Odonata, vários estudos têm demonstrado que a diversidade desse grupo é afetada em uma escala local principalmente por modificações da vegetação (SAMWAYS et al., 1996; CARVALHO et al., 2013; OLIVEIRA-JUNIOR et al., 2017).

Com os resultados obtidos neste trabalho, podemos observar que os valores do IIH exercem efeito significativo sobre as subordens de Odonata. O aumento do nível de integridade do habitat exerce efeito positivo sobre a abundância e riqueza de Zygoptera; porém, este mesmo efeito é negativo em Anizoptera, pois as especificidades ecofisiológicas de anisóptera são mais voltadas para áreas abertas. Corroborando com a hipótese de que a subordem Anisoptera é mais tolerante a alterações no ambiente do que os Zygoptera (MARCO, 1998; FERREIRA-ROMERO, 1998).

O percentual de cobertura de dossel também exerceu efeito significativo sobre as duas subordens quando comparadas. A comunidade de Anisoptera apresentou declínio em sua abundância e riqueza em ambientes com maior percentual de cobertura de dossel, já a comunidade de Zygoptera apresentou aumento na abundância e riqueza nos mesmos ambientes.

A retirada de vegetação ciliar e o aumento dos raios solares são benéficos a comunidade de Anisoptera proporcionando aumento na riqueza de espécies. Por serem heliotérmicos e pousadores, a sua abundância é dependente da radiação solar (REMSBURG et al., 2008), necessária para fornecer calor para seus corpos e iniciarem suas atividades (MAY, 1976; CORBET et al., 2008; RESENDE, 2010). Desta forma, indivíduos dessa subordem geralmente apresentam maior abundância e maior riqueza em áreas mais abertas, com menor cobertura de dossel (MAY, 1991; MAY, 1979). Isso sugere que a abundância dos indivíduos diminui conforme a intensidade de sombra aumenta (KINVIG et al., 2000; SAMWAYS et al., 2004; REMSBURG et al.,2008).

Os Odonata de maior porte (grande parte dos Anisoptera) apresentam grande capacidade de dispersão, o que pode levar a uma maior distribuição geográfica (COBERT, 1999) e consequentemente terem capacidade de alcançarem ambientes com maior incidência de luz solar. Indivíduos da subordem Anisoptera geralmente são beneficiados pela alteração das áreas naturais em função de suas habilidades de termorregulação, pois tendem a apresentar mecanismos homeostáticos mais eficientes e maior mobilidade, tolerando condições ambientais mais diversificadas (HUGHES et al., 2000; TSCHARNTKE et al., 2002). A riqueza desta subordem em ambientes alterados também pode ter sido provocada pela proximidade de ambientes lênticos, possibilitando que os insetos dispersassem para os igarapés amostrados nesta categoria (MARCO et al., 1998; CARVALHO et al., 1998).

A riqueza e abundância de Zygoptera se apresentam altas em ambientes preservados, com presença de vegetação ciliar, por possuir necessidades biológicas específicas (COBERT, 1999), sendo pequenas e com corpo delgado, é possível que, em virtude da sua alta razão superfície volume, sejam mais sujeitas ao 
superaquecimento e à dessecação, tornando-se mais sensíveis às variações ambientais por restrições ecofisiológicas (CORBET, 1999; CORBET et al., 2008), suas temperaturas variam de acordo com a temperatura do ambiente, sendo assim associados a ambientes com maior quantidade de cobertura vegetal (MAY, 1976; COBERT, 1999).

A abertura de dossel é benéfica a Anisoptera e causa uma redução na diversidade de Odonata como um todo, visto que os Zygoptera por sua baixa capacidade de dispersão no ambiente, não serão encontradas ou diminuirão nessas áreas (JUEN et al., 2011), pois são mais dependentes das condições locais com áreas mais cobertas por vegetação (menor incidência de radiação solar) (MARCO et al., 2002; MAY, 1991; MAY, 1979; MARCO et al., 2005). Em áreas onde a abertura no dossel ocorre de forma natural, ocorre um aumento na biodiversidade de Odonata, pois possibilita que as duas subordens coexistam.

\section{CONCLUSÕES}

A diferença na distribuição dos indivíduos nos ambientes estudados pode ser entendida através de suas limitações fisiológicas. De um modo geral, demonstramos que a vegetação ripária é o fator que mais afeta tanto a riqueza de espécies da subordem Anisoptera, quanto a de Zygoptera em riachos tropicais, embora outros fatores também possam afetar a diversidade de Odonata. Por este motivo, podemos afirmar que Anisoptera, por depender da luz solar e possuir maior poder de dispersão no ambiente, são mais resistentes a mudanças em seu hábitat. Zygoptera, devido a possuir menor porte corpóreo, apresenta limitações de dispersão nos ambientes, e por não poder sofrer intensa incidência da radiação solar, são cotadas como mais sensíveis a mudanças em seu habitat, e, portanto, os Anisoptera são bons indicadores de áreas alteradas, e os Zygoptera de áreas preservadas.

\section{REFERÊNCIAS}

ANGERMEIER, P. L.; KARR, J. R.. Biological integrity versus biological diversity as policy directives. BioScience, v.44, p.690-697, 1994. DOI: http://doi.org/10.1007/978-1-46124018-1

BAPTISTA, D. F.; DORVILLÉ, L. F. M.; BUSS, D. F.; NESSIMIAN, J. L.. Spatial and temporal organization of aquatic insects assemblages in the longitudinal gradient of a tropical river. Brazilian Journal of Biology, São Carlos, v.61, p.295-394, 2001. DOI: http://doi.org/10.1590/S0034$\underline{71082001000200012}$

BELLE, J. A.. Synopsis of the species of Phyllocycla Calvert with description of four new taxa and a key to the genera of the neotropical Gomphidae (Odonata, Gomphidae). Tijdschrift voor Entomologie, v.131, p.73-102, 1998.

BELLE, J.. Higher classification of the South-American Gomphidae (Odonata). Zoologische Mededelingen, v.70, p.298-324, 1996.

BORROR, D. J.. A key to the New World genera of Libellulidae (Odonata). Annals of the Entomological Society of America, v.38, p.168-94, 1945. DOI:

http://doi.org/10.1093/aesa/38.2.168
CALLISTO, M.; MORENO, P.. Bioindicadores como ferramenta para o manejo, gestão e conservação ambiental. In: SIMPÓSIO SUL DE GESTÃO E CONSERVAÇÃO AMBIENTAL 'SOCIEDADE E SUSTENTABILIDADE', 2. Anais. Erechim: URI, 2006.

CALLISTO, M.; MORETTI, M.; GOULART, M..

Macroinvertebrados bentônicos como ferramenta para avaliar a saúde de riachos. Revista Brasileira de Recursos Hídricos, Porto Alegre, v.6, p.71-82, 2001. DOI: http://doi.org/10.21168/rbrh.v6n1.p71-82

CARVALHO, A. L.; NESSIMIAN, J. L.. Odonata do Estado do Rio de Janeiro, Brasil: Hábitats e hábitos das larvas. In: NESSIMIAN, J. L.; CARVALHO, A. L.. Ecologia de Insetos Aquáticos. Rio de Janeiro: UFRJ, 1998. p.309.

CARVALHO, F. G.; SILVA-PINTO, N.; OLIVEIRA-JUNIOR, J. M. B.; JUEN, L.. Effects of marginal vegetation removal on Odonata communities. Acta Limnologica Brasiliensia, v.25, p.10-18, 2013. DOI: http://doi.org/10.1590/S2179-975X6114

CLAUSNITZER, V.; KALKMAN, V. J.; RAM, M.; COLLEN B.; COLLEN, B.; BAILLIE, J. E.; BEDJANIČ, M.; DARWALL, W. R.; DIJKSTRA, K. D. B.; DOW, R.; HAWKING, J.; KARUBE, H.; MALIKOVA, E.; POULSON, D.; SHÜTTE, K.; SUHLING, F.; VILLANUEVA, R. J.; ELLEMIEDER, N.; WILSON, K.. Odonata 
enter the biodiversity crisis debate: The first global assessment of an insect group. Biological Conservation, v.142, n.8, p.1864-1869, 2009. DOI:

https://doi.org/10.1016/j.biocon.2009.03.028

CORBET, P. S.. Dragonflies: Behaviour and Ecology of Odonata. London: Comstock Publishing Associates, 1999.

CORBET, P. S.. A Biology of Dragonflies. London: Classey, 1983.

CORBET, P. S.; DUNKLE, S. W.; UBUKATA, H.. Proceeding of International Symposium on the conservation of dragonflies and their habists. Kushiro: SPB, 1995. DOI: http://doi.org/10.1126/science.1208742

CORBET, P. S.; MAY, M. L.. Fliers and perchers among Odonata: dichotomy or multidimensional continuum: a provisional reappraisal. International Journal of Odonatology, v.11, p.155-171, 2008. DOI: http://doi.org/10.1080/13887890.2008.9748320

ESPÍRITO-SANTO, F. D. B.. Caracterização e mapeamento da vegetação da região da Floresta Nacional do Tapajós através de dados óticos, radar e inventários florestais. Tese (Doutorado) - Instituto Nacional de Pesquisas Espaciais, São José dos Campos, 2003.

FERREIRA-PERUQUETTI, P. S. F.. Odonata (libélulas) do município de Luís Antônio, São Paulo, Brasil: Reação com o solo e riqueza faunística. Tese (Doutorado em Ecologia e Recursos Naturais) - Universidade Federal de São Carlos, São Carlos, 2004.

FERREIRA-PERUQUETTI, P. S.; MARCO, P. J.. Efeito da alteração ambiental sobre a comunidade de Odonata em riachos de Mata Atlântica de Minas Gerais, Brasil. Revista Brasileira de Zoologia, Curitiba, v.19, p.317-327, 2002. DOI: http://doi.org/10.1590/S0101-81752002000200002

FERREIRA-ROMERO, M.. New data on the ecological tolerance of some Rheophilous Odonata in Mediterranean Europe (Sierra Morena, Southern Spain). Odonatologica, v.17, p.121-126, 1998.

FIRMINO, W. G.. Análise do Impacto da Ação Antrópica na Microbacia do Córrego Lava-Pés em Ipameri - Goiás. Monografia (Graduação) - Universidade Estadual de Goiás, Goiás, 2003.

FULAN, J. A.; HENRY, R.. Temporal distribution of immature Odonata (Insecta) on Eichhorniaazurea (Kunth) stands in the Camargo Lake, Paranapanema River, São Paulo. Revista Brasileira de Entomologia, Curitiba, v.51, p.224-227, 2007.

GALVÃO, A. M. P.. Reflorestamento de propriedades rurais para fins produtivos e ambientais: um guia para ações municipais e regionais. Brasília: Embrapa, 2000.

GARRISON, R. W.. A synopsis of the genus Hetaerina with descriptions of four new species (Odonata: Calopterigidae). Revista Brasileira de Entomologia, Curitiba, v.116, p.175259, 1990.

GARRISON, R. W.; ELLENRIEDER, N.; LOUTON, J. A.. Damselfly Genera of the New World: An Illustrated and Annotated Key to the Zygoptera. The Johns Hopkins University Press, Baltimore, p.490, 2010. DOI: http://doi.org/10.1653/024.093.0434

GIEHL, N. F. S.; DIAS-SILVA, K.; JUEN, L.; BATISTA, J. D.; CABETTE, H. S. R.. Taxonomic and numerical resolutions of Nepomorpha (Insecta: Heteroptera) in Cerrado streams. PLoSONE, v.9, n.8, 2014. DOI: https://doi.org/10.1371/journal.pone.0103623

GORHAM, C.; VODOPICH, D.. Effects of acidific $\mathrm{pH}$ on predation rates and surviorship of damselfly nymphs. Hydrobiologia, v.242, p.51-62, 1992.

HUGHES, T. P.; BAIRD, A. H.; DINSDALE, E. A.; MOLTSCHANIWSKYJ, N. A.; PRATCHETT, M. S.; TANNER, J. E.; WILLIS, B. L.. Supply-side ecology works both ways: The link between benthic adults, fecundity, and larval recruits. Ecology, v.81, p.2241-2249, 2000. DOI: http://doi.org/10.2307/177111

JUEN, L.; MARCO, P. J.. Odonate beta diversity in terra-firme forest streams in Central Amazonia: On the relative effects of neutral and niche drivers at small geographical extents. Insect Conservation and Diversity, v.4, p.265-274, 2011. DOI: https://doi.org/10.1111/j.1752-4598.2010.00130.x

KINVIG, R. G.; SAMWAYS, M. J.. Conserving dragonflies (Odonata) along streams running through commercial forestry. Odonatologica, v.29, p.195-208, 2000.

LENCIONI, F. A. A.. The Damselflies of Brazil: An Illustrated Guide-the non Coenagrionidae Families. São Paulo: All Print, 2005.

MARCO, P. J., LATINI, A. O.; RESENDE, D. C..

Thermoregulatory constraints on behavior: patterns in a Neotropical dragonfly assemblage. Neotropical Entomology, v.34, p.155-162, 2005. DOI: http://doi.org/10.1590/S1519$\underline{566 \times 2005000200002}$

MARCO, P. J.; LATINI, A. O.. Estrutura de guildas e riqueza de espécies em uma comunidade de larvas de Anisoptera (Odonata). In: NESSIMIAN, J. L.; CARVALHO A. L.. Ecologia de insetos aquáticos. Rio de Janeiro: UFRJ, 1998. p.101-112

MARCO, P. J.; RESENDE, D. C.. Activity patterns and thermoregulations in a tropical dragonflies assemblage. Odonatalogica, v.31, n.31, p.129-138, 2002.

MARCO, P.. The Amazonian Campina dragonfly assemblage: patterns un microhabitat use and behaviour in a foraging habitat (Anisoptera). Odonatologica, v.27, p.239-248, 1998.

MATSUURA, K.. Bioindicadores em Ecossistemas. Paris: Unesco, 2000.

MATTHEWS, R. A.; BUIKEMA, A. L.; CAIRNS, J.. Biological monitoring part IIA: Receiving, system functional methods relationships, and indices. Water Research, v.16, p.129-139, 1982. DOI: http://doi.org/10.1016/0043-1354(82)90102-6

MAY, M. L.. Insect thermoregulation in adaptation. Annual Review of Entomology, v.24, p.313-349, 1979. DOI: http://doi.org/10.1146/annurev.en.24.010179.001525

MAY, M. L.. Thermal adaptation of dragonflies, revisited. Advanced Odonata, v.5, p.71-88, 1991.

MAY, M. L.. Thermoregulation in adaptation to temperature 
in dragonflies (Odonata: Anisoptera). Ecological

Monographs, v.46, p.1-32, 1976. DOI:

http://doi.org/10.2307/1942392

MONTEIRO-JÚNIOR, C. S.; COUCEIRO, S. R. M.; HAMADA, N.; JUEN, L.. Effect of vegetation removal for road building on richness and composition of Odonata communities in Amazonia, Brazil. International Journal of Odonatology, v.16, p.135-44, 2013. DOI:

https://doi.org/10.1080/13887890.2013.7647

MONTEIRO-JÚNIOR, C. S.; JUEN, L.; HAMADA, N.. Effects of urbanization on stream habitats and associated adult dragonfly and damselfly communities in central Brazilian Amazonia. Landscape Urban Plan, v.127, p.28-40, 2014. DOI: http://doi.org/10.1016/j.landurbplan.2014.03.006

NESSIMIAN, J. L.; VENTICINQUE, E.; ZUANON, J.; MARCO, P. J.; GORDO, M.; FIDELIS, L.; BATISTA, J. D.; JUEN, L.. Land use, habitat integrity, and aquatic insect assemblages in Central Amazonian streams. Hydrobiologia, p.117-131, 2008. DOI: http://doi.org/10.1007/s10.750-008-9441-x

OLIVEIRA-JUNIOR, J. M. B.; DE MARCO, P.; DIAS-SILVA, K.; LEITÃO, R. P.; LEAL, C. G.; POMPEU, P. S.; GARDER, T. A.; HUGHES, R. M.; JUEN. L.. Effects of human disturbance and riparian conditions on Odonata (Insecta) assemblages in eastern Amazon basin streams. Limnologica, v.66, p.31-39, 2017. DOI: https://doi.org/10.1016/j.limno.2017.04.007

OLIVEIRA-JUNIOR, J. M. B.; SHIMANO, Y.; GARDNER, T. A.; HUGHES, R. T. M.; MARCO, P.; JUEN, L.. Neotropical dragonflies (Insecta: Odonata) as indicators of ecological condition of small streams in the eastern Amazon. Amazon Austral Ecology, v.40, p.733-744, 2015. DOI:

http://doi.org/10.1111/aec.12242

PEREIRA, L. R.; CABETTE, H. S. R.; JUEN, L.. Trichoptera as bioindicators of habitat integrity in the Pindaíba River Basin, Mato Grosso (Central Brazil). Annales de Limnologie, v.48, p.295-302, 2012. DOI: http://doi.org/10.1051/limn/2012018

POND, G. J.; PASSMORE, M. E.; BORSUK, F. A.; REYNOLDS, L.. Downstream effects of mountaintop coal mining: comparing biological conditions using family-and genus-level macroinvertebrate bioassessment tools. Journal of the North American Benthological Society, v.27, p.717-737, 2008. DOI: http://doi.org/10.1899/08-015.1

REMSBURG, A. J.; OLSON, A. C. SAMWAYS, M. J.. Shade alone reduces adult dragonfly (Odonata: Libellulidae) abundance. Journal Insect Bahaviour, v.21, p.460-468, 2008. DOI: http://doi.org/10.1007/s10905-008-9138-z
RESENDE, D. C.. Residence advantage in heterospecific territorial disputes of Erythrodiplax Brauer species (Odonata, Libellulidae). Revista Brasileira de Entomologia, São Paulo. v.54, p.110-114, 2010. DOI: http://doi.org/10.1590/S0085$\underline{56262010000100014}$

SAMWAYS, M. J.; STEYTLER, N. S.. Dragonfly (Odonata) distribution patterns in urban and forest landscapes, and recommendations for riparian management. Biological Conservation, v.78, p.279-288, 1996. DOI: http://doi.org/10.1016/S0006-3207(96)00032-8

SAMWAYS, M. J.; TAYLOR, S.. Impacts of invasive alien plants on Red-Listed South African dragonflies (Odonata). South African Journal of Science, v.100, p.78-80, 2004.

SANTOS, M. B. L.; ROCHA, L. A.; MARQUES, M. M. G. S. M.; BARBOSA, F. A. R.. Diversidade e abundância da fauna bentônica de cinco lagoas do Karste do planalto de Lagoa Santa, Minas Gerais. Oecologia Brasiliensis, Rio de Janeiro, v.5, p.77-89, 1998.

SOUZA, L. O. I.; COSTA, J. M.; OLDRINI, B. B.. Odonata. In: FROEHLICH, C. G.. Guia on line: Identificação de larvas de Insetos Aquáticos do Estado de São Paulo. São Paulo: 2007.

SOUZA, T. S.; FONTANETTI, C. S.. Ensaio do Cometa para Avaliação da Qualidade das Águas do Rio Paraíba do Sul, numa área sob influência de uma Refinaria de Petróleo. In: CONGRESSO BRASILEIRO DE PESQUISA E DESENVOLVIMENTO EM PETRÓLEO E GÁS. Anais. Campinas: 2007.

TSCHARNTKE, T.; STEFFAN-DEWENTER, I.; KRUESS, A.; THIES, C.. Contribution of small habitat fragments to conservation of insect communities of grassland - cropland landscapes. Ecological Applications, v.12, p.354-363, 2002. DOI:

http://doi.org/10.1890/1051 0761(2002)012[0354:COSHFT]2.0.CO;2

VALENTE, O. F.; GOMES, M. A.. Conservação de Nascentes: Hidrologia e Manejo de Bacias Hidrográficas de Cabeceiras. Viçosa: Aprenda Fácil, 2005.

VEECH, J. A.; SUMMERVILLE, K. S.; CRIST, T. O.; GERING, J. C. The additive partitioning of species diversity: recent revival of an old idea. Oikos, v.99, p.3-9, 2002. DOI: http://doi.org/10.1034/j.1600-0706.2002.990101.x

ZAR, J. H.. Biostatistical analysis. 5 ed. New Jersey: PrenticeHall Press, 2008.

ZAR, J. H.. Biostatistical analysis. New York: Prentice-Hall Press, 1999.

A CBPC - Companhia Brasileira de Produção Científica (CNPJ: 11.221.422/0001-03) detém os direitos materiais desta publicação. Os direitos referem-se à publicação do trabalho em qualquer parte do mundo, incluindo os direitos às renovações, expansões e disseminações da contribuição, bem como outros direitos subsidiários. Todos os trabalhos publicados eletronicamente poderão posteriormente ser publicados em coletâneas impressas sob coordenação da Sustenere Publishing, da Companhia Brasileira de Produção Científica e seus parceiros autorizados. Os (as) autores (as) preservam os direitos autorais, mas não têm permissão para a publicação da contribuição em outro meio, impresso ou digital, em português ou em tradução. 